\title{
Influence of the Distance Between Nozzle and Substrate on Structural, Photoluminescence, and Detector Characteristics of p- NiO/n-Si Hetero-Junction Deposited by Spray Pyrolysis Method
}

Husam R. Abed ( $\square$ hussamrafat@yahoo.com)

Iraq Ministry of Education https://orcid.org/0000-0002-2728-6606

Ameer I. Khudadad

Iraq Ministry of Education

Fadhil Mahmood Oleiwi

Iraq Ministry of Education

\section{Research Article}

Keywords: NiO, UV Photodetector, Spray pyrolysis, Porous microstructures.

Posted Date: December 20th, 2021

DOI: https://doi.org/10.21203/rs.3.rs-1184439/v1

License: @ (i) This work is licensed under a Creative Commons Attribution 4.0 International License. Read Full License 


\section{Abstract}

In the present investigation, $\mathrm{p}-\mathrm{NiO}$ has been deposited on $\mathrm{n}$-Si (100) substrate by the spray pyrolysis method. The effect of the distance between the substrate and the nozzle on the structural, photoluminescence, and detection properties has been well inspected. XRD analysis proved the polycrystalline system with a cubic structure for $\mathrm{NiO}$. The elemental analysis confirmed the existence of $\mathrm{Ni}, \mathrm{O}$, and $\mathrm{Si}$ materials without any impurities. The FESEM analysis showed nano and micro particles distributed on the Si layer, the micro particles have porous like structures which play a significant role as photons guider. The photoluminescence measurement depicted three main peaks at the UV and visible regions of the electromagnetic spectrum which are related to near band edge emission and defects within the crystal, respectively. I-V characteristics revealed good conductivity under UV illumination, and the highest current was recorded by a sample when the distance between the nozzle and the substrate is $25 \mathrm{~cm}$. The responsivity elucidated a high value at UV region with 6.5 $\mathrm{mA} / \mathrm{W}$, and the current-time properties demonstrated good reproducibility, high stability and photoresponse, and rapid response and recovery times of 0.375 and $0.791 \mathrm{~s}$, respectively at a lower bias voltage of 1.5 Volt under UV photons source.

\section{Introduction}

The enhancement of Ultra-Violet (UV) photodetectors is very significant, this is due to its employment in many fields such as missile tracing, optical communication, chemical and biological sensors, image sensing, and flame detection [1, 2]. Many researchers have used metal oxide semiconductors due to their wide band gap, ease to fabricate, and low cost materials [3-5]. Some scientists have deposited these materials on $\mathrm{Si}$, glass, alumina, sapphire, or quartz substrates and used them for various applications [6-9]. The enhancement process of UV photo detectors should involve response time, restoration time, responsivity, and detectivity [10-12]. For this purpose, numerous articles have tried to improve the performance of the detectors using metal oxide semiconductors such as $\mathrm{ZnO}, \mathrm{TiO}_{2}, \mathrm{NiO}$, $\mathrm{SnO}_{2}$, and $\mathrm{WO}_{3}$ [13-17]. Among these materials, Nickel Oxide (NiO) is the preferred one, the reason behind the selection of this p-type metal oxide semiconductor is related to its direct and wide energy band gap, high surface area, high detectivity and responsivity, easy to fabricate, and low cost device [18-20]. The perfect technique to improve the detector's characteristics is the fabrication of P-N heterojunction [21-24]. The creation of $\mathrm{p}-\mathrm{Ni} / \mathrm{n}-\mathrm{Si}$ hetero-junction leads to reduction of leakage current because of establishing a strong electric field at the interface between $\mathrm{Ni}$ and $\mathrm{Si}$ and in turn develop the photocurrent and the restoration time [25]. Therefore, it is significant to use this hetero-junction for detecting a wide range of UV and visible light in the spectrum region. Choi et al [26] have evaporated $\mathrm{NiO}$ on a substrate of silicon, and their hetero-junction was response for visible and UV regions. Zhang et al [27] have fabricated a photodetector based on $\mathrm{p}-\mathrm{NiO} / \mathrm{n}-\mathrm{Si}$ hetero-junction. This detector was created by UV oxidation of $\mathrm{Ni}$, and it showed a low leakage of current density in comparison with that fabricated by the sputtering method. These detectors have slow response time, low EQE, and low photo current and dark current, this is due to the amorphous structure of $\mathrm{NiO}$ which is reducing the lifetime of the carrier. There are several techniques for NiO thin films deposition such as pulsed laser deposition [28], spray pyrolysis technique [29], chemical bath deposition [30], sputtering [31], and spin coating method [32]. Among these methods, the spray pyrolysis method is low cost, easy to prepare the film, high purity product, obtained homogeneous film, high chemical nucleation reaction, and thickness of the film well controlled through this method. According to an extensive search, there are few articles that deal with the deposition of $\mathrm{NiO}$ by spray pyrolysis method on Si substrate and then study its detector characteristics. The aim of this work is to fabricate a UV detector based on the spray pyrolysis method of $\mathrm{NiO}$ on Si substrate and study the effect of distance between the nozzle and the substrate on structural, photoluminescence, and detector properties.

\section{Experimental Work}

Before of deposition method, $1 \mathrm{~cm}^{2}$ silicon substrates of (100) orientation, n-type, and 1-5 $\Omega . \mathrm{cm}$ resistivity have well cleaned by washing them in ethanol and distilled water. After the cleaning, a spray pyrolysis system was provided for the deposition process and it can be seen in [33]. The starting material is $0.2 \mathrm{M}$ of $\mathrm{NiCl}_{2} .2 \mathrm{H}_{2} \mathrm{O}$ (99.9\% purity from Changsha Easchem Co. Ltd., China), this material was dissolved in $70 \mathrm{ml}$ of $99.99 \%$ ethanol solution in a $100 \mathrm{ml}$ beaker. Then, this solution was carried out into a magnetic stirrer for $25 \mathrm{~min}$ at the room temperature, and the obtained solution is greenish. The final solution was used in the spray pyrolysis system. The based conditions of the spray process are as follow: the temperature of the deposition was fixed at $375^{\circ} \mathrm{C}$, the pressure of the nitrogen gas was fixed at 2.4 bar, the spraying time was $6 \mathrm{~s}$ while the duration between two sprays was $80 \mathrm{~s}$ to keep the temperature at its constant degree and to give the chance for more nucleation reactions, and the distance between the nozzle and the sample was varied $(20,25$, and $30 \mathrm{~cm}$ ) in order to select the optimized distance for deposition process and its effect on the NiO/Si characteristics. Each film was deposited with $50 \mathrm{ml}$ with the former solution. 


\section{Characterizations}

The structural properties were investigated by X-Ray diffractometer instrument (6000-SHIMADZU) with Cu Ka source $(0.15406 \mathrm{~nm}$ wavelength) and the scanning $2 \theta$ is in range of $\left(30-70^{\circ}\right)$. The surface morphology was inspected by field emission scanning electron microscope (FESEM) with a high resolution image (T EASCAN manufactured by a company (UEGA. LM), Geck origin), and the chemical element was confirmed by electron dispersive X-ray (EDX) analysis along with FESEM instrument. Photoluminescence (PL) (Hitachi 3100 ) with an exciting wavelength of $325 \mathrm{~nm}$ at the room temperature was used to investigate the emission spectra of the samples. The current-voltage characteristics of the hetero-junction were inspected by Hewlett Packard 34401A multimeter and Keithley 6517A Electrometer/High Resistance Meter with the voltage sweeps from $-1.5 \mathrm{~V}$ to $+1.5 \mathrm{~V}$. The current-time properties were proved under dark and illumination case using $365 \mathrm{~nm}$ LED at a constant voltage of $1.5 \mathrm{~V}$. The thickness of the film was measured by a laser reflection method in which a $632 \mathrm{~nm}$ He-Ne laser was used, and the thickness of the films that deposited at 20, 25, and $30 \mathrm{~cm}$ distance were 463, 457, and 471, respectively. The schematic of the photodetector device and the electrode configuration is depicted in Figure 1.

\section{Results And Discussion XRD analysis}

The purpose of using X-Ray diffract-meter analysis is to identify the employed materials in the work, and after confirming the materials one can determine the other related parameters. The XRD patterns of $\mathrm{NiO} / \mathrm{Si}$ at different distances between the nozzle and the substrate have been investigated and depicted in Figure 2. In this figure, it could be observed that there are three main peaks at 2 thetas of 37.28 , 43.38 , and $62.98^{\circ}$ which are corresponding to the main planes of (111), (200), and (220) respectively. All of the patterns showed a polycrystalline system with a cubic structure. Also, from the figure, one can notice that the intensity of the detected peaks has increased when the distance between the nozzle and the substrate increases from 20 to $25 \mathrm{~cm}$, whereas it has decreased when the distance increases to $30 \mathrm{~cm}$. The obtained patterns are matched with those prior reported [34-38]. The increase in intensity leads to a high degree of crystallinity. The crystal size, the dislocation density, and the micro strain were calculated and tabulated in Table 1 . They were calculated by the following formulas [39-41]:

$$
D=\frac{k \times \lambda}{\beta \times \cos \theta}
$$

1

$$
\delta=\frac{1}{D^{2}}
$$

2

$$
\text { Microstrain }=\frac{\beta \cos \theta}{4}
$$

3

Where, $k$ is the shape factor with a value of $0.9, \lambda$ is the wavelength of the radiation, $\beta$ is the full width at half maximum, $\theta$ is Bragg's angle,

From Table 1, it is observed that the crystal size increased when the distance between the substrate and the nozzle has increased from 20 to $25 \mathrm{~cm}$. This leads to high crystallinity and nucleation processes. Whereas, it has decreased when the distance increased to $30 \mathrm{~cm}$. Moreover, the dislocation density and the micro strain decreases, and the optimum value was found at the distance of $25 \mathrm{~cm}$, in which the defects have decreased. The crystal size and the dislocation density have been well monitored, and the reason behind this trend is linked to the distance between the nozzle and the substrate which is the main factor that impacts the size of the crystals and hence improving the degree of crystallization. In the case of the distance $20 \mathrm{~cm}$, the droplet is large and when it reaches the substrate there is no enough temperature to vaporize the droplet, hence it will be a weak cohesion of the film on the substrate. In the case of $30 \mathrm{~cm}$, the droplet is small and it is vaporized before it reaches the substrate, hence when it touches the substrate, it will transform to powder. Whereas in the case of $25 \mathrm{~cm}$, the droplets with an optimum size reach the substrate and evaporate at the moment before they touch the substrate, then it will be a good film with high cohesion with the substrate [42, 43]. Therefore, it is very important to adjust the distance between the nozzle and the substrate.

Page 3/16 
Table 1

XRD parameters of $\mathrm{NiO} / \mathrm{Si}$ at different distances between the nozzle and the Si substrate.

\begin{tabular}{|llllll|}
\hline $\begin{array}{l}\text { Distance between nozzle and } \\
\text { substrate (cm) }\end{array}$ & $\begin{array}{l}\text { 20 of (111) main plane } \\
\text { (deg.) }\end{array}$ & $\begin{array}{l}\text { FWHM } \\
(\mathbf{d e g})\end{array}$ & $\begin{array}{l}\text { Crystal size } \\
(\mathbf{n m})\end{array}$ & $\begin{array}{l}\text { Dislocation density } \\
\left(\mathbf{n m}^{-2}\right)\end{array}$ & $\begin{array}{l}\text { Micro } \\
\text { strain }\end{array}$ \\
\hline 20 & 37.28 & 0.3 & 27.94 & 0.1891 & 0.00124 \\
\hline 25 & 37.28 & 0.22 & 38.1 & 0.1619 & 0.00091 \\
\hline 30 & 37.28 & 0.26 & 32.24 & 0.1761 & 0.00107 \\
\hline
\end{tabular}

FESEM analysis

The suitable method to analyze the surface morphology of thin film is field emission scanning electron microscopy (FESEM) in which the beam of electrons accelerated with high voltage toward the film. The interaction between the accelerated electrons and the electrons of the target materials leads to scattering of electrons, the scattered electrons will be detected by a detector which will plot the morphology of the target materials. The surface morphology of Ni deposited on Si substrate at different distances between the nozzle and the substrate is illustrated in Figure 3. Figure 3a shows the morphology of Ni/Si deposited at a distance of $20 \mathrm{~cm}$, this figure depicted microstructure particles and under them nanostructure particles, these particles covered the silicon layer, this is due to the small distance between the nozzle and the substrate that makes the particles aggregated and covered the substrate. For Figure $3 \mathrm{~b}$ (the distance between the nozzle and the substrate is $25 \mathrm{~cm}$ ), it manifests porous microstructure particles which not covered all the silicon layer, there are spaces between these microstructures that contain small nanoparticles and parts of the silicon layer are exposed. These large porous like structures in microparticles will be a good guider for the incident photons in order to enhance the detector performance. When the distance between the nozzle and the substrate is $30 \mathrm{~cm}$, as in Figure 3c, it demonstrates large exposure areas (not covered), furthermore on the right side of the figure there is a big and bright microstructure, the brightness is due to the high resistance of target material which will slow the speed of the accelerated electrons and then these electrons will interact with the other accelerated beam, this interaction will appear as high brightness. Some articles have also obtained microstructure particles of NiO, [44] have found agglomerated NiO microstructures flaks. [45] have found micro spherical NiO particles, mesoporous cubic, and hexagonal nanocrystal. [46] have found micro platelets morphology. [47] have obtained Vanadium doped Ni microstructures of random shapes. [48] have found micropatterns structures of $\mathrm{NiO} / \mathrm{Cr}$ fabricated by femtosecond laser reductive.

\section{Edx Analysis}

This measurement is typically used to specify the chemical elements and their weights. The chemical elements analysis of Ni/Si that deposited at different distances between the nozzle and the substrate is shown in Figure 4. This figure confirms the presence of Ni, $\mathrm{O}$, and Si chemical elements without any other unwanted materials. When the distance between the nozzle and the substrate increases from 20 to $25 \mathrm{~cm}$, as in Figure $4 \mathbf{a}$ and b, the weight of the Ni element increases about $3 \%$, the weight of the 0 element decreases by about $5 \%$, and the weight of Si element increases about $2 \%$. The rising in weight of the Ni element leads to a high crystallization process, while the reduction in weight of the 0 element is related to the reduction in defects (as proved from XRD analysis), and the rising in weight of the Si element is linked to the coalescence of nanoparticles and give the opportunity for silicon layer to appear in some regions. Whereas the film that deposited on Si substrate by a nozzle with a distance of $30 \mathrm{~cm}$ from the substrate (Figure $4 \mathrm{c}$ ) demonstrates the lowest percentage of Ni element, a higher percentage of $\mathrm{O}$ element than that in Figure $4 \mathrm{~b}$, and the highest percentage of Si element.

\section{Photoluminescence}

The photoluminescence of $\mathrm{NiO} / \mathrm{Si}$ was tested at the room temperature with the excitation wavelength source of $325 \mathrm{~nm}$. Figure 5 reveals the PL spectra of NiO deposited on Si substrate at various distances between the substrate and the nozzle. This figure depicts that there are three main peaks related to the UV and Visible regions of the electromagnetic spectrum. The peak at the UV zone is linked to the near band edge emission of $\mathrm{NiO}$ which is influenced by the arrangement of stoichiometric of anions and cations. This peak is located at around $366.68 \mathrm{~nm}$ for the distances of 20 and $30 \mathrm{~cm}$, which is corresponding to $3.38 \mathrm{Ev}$ of energy band, while it is located at $368 \mathrm{~nm}$ for the distance of $25 \mathrm{~cm}$, which is corresponding to $3.36 \mathrm{Ev}$. The other peak is positioned at the visible zone that is connected to the

Page $4 / 16$ 
defects within the $\mathrm{NiO}$ crystal such as oxygen vacancies, oxygen interstitial, and $\mathrm{Ni}$ interstitial, these defects could act as the trappers for the electrons $[49,50]$.

The other two peaks are located at $442 \mathrm{~nm}$ and $532 \mathrm{~nm}$ (visible region) for the films that deposited on a silicon substrate with distances 20 and $30 \mathrm{~cm}$, these peaks are corresponding to 2.8 and $2.33 \mathrm{Ev}$. However, the peaks of the film that deposited on a silicon substrate with a distance of $25 \mathrm{~cm}$ are located at 446 and $534 \mathrm{~nm}$ which are corresponding to 2.78 and $2.32 \mathrm{Ev}$, respectively.

From the above description, one can note that there is a small shift towards the high value of wavelength when the film is deposited with a distance of $25 \mathrm{~cm}$, this is related to the slight increase in grain size of the structure.

\section{Current-Voltage characteristics}

The current-voltage characteristics of $\mathrm{p}-\mathrm{NiO} / \mathrm{n}-\mathrm{Si}$ are shown in Figure 6. This detector was tested under dark and illuminated with a blue light source (Light Emit Diode) with a power of $2 \mathrm{Mw}$ and $365 \mathrm{~nm}$ wavelength. The performance of P-Ni/n-Si in the dark case shows rectifying trend which improves the creation of the p-n junction at the interface between the p-Ni and the n-Si, as in Figure 6 a. The photodetector depicted an improved photocurrent under the illumination of the blue light source. In this case, the blue light motivates the material to generate electron-hole pairs. At the same time, the electric field has been built at the interface by the applied reverse bias voltage, which serves as an electromotive force to transfers the electron to the silicon region and transfers the holes to the Ni region, Figure 6b. Furthermore, it can be noticed that, when the Si substrate was sprayed by $\mathrm{Ni}$ at a distance of $25 \mathrm{~cm}$, it showed good rectifying behavior under dark and illumination conditions. This is related to the large width of the depletion layer, large grain size, and a high degree of stoichiometry [51], also, the increase of crystal size gives chance to the reduction of the scattering process by charge carriers [52]. Moreover, the rising of photocurrent with the augmentation of voltage value is due to the rising in carrier drift velocity [53].

\section{Detector Properties}

The responsivity of $\mathrm{Ni} / \mathrm{Si}$ at a bias voltage of $1.5 \mathrm{~V}$ and wavelength range of 300-900 $\mathrm{nm}$ at a constant power of $2 \mathrm{~mW}$ for the sample that deposited at $25 \mathrm{~cm}$ distance between the nozzle and the substrate is shown in Figure 7. There are two main peaks for responsivity, one of them is sharp, high intensity, in the UV-region of the electromagnetic spectrum, and the responsivity is around $6.5 \mathrm{~mA} / \mathrm{W}$. The other peak is broad, low intensity, at the NIR region of the electromagnetic spectrum, and the responsivity is around $3.4 \mathrm{~mA} / \mathrm{W}$. The reason behind the presence of two regions for the responsivity is related to the existence of two materials $\mathrm{Ni}$ and $\mathrm{Si}$, the $\mathrm{Ni}$ is response to UV light, while $\mathrm{Si}$ is response to NIR light. Subsequently, the higher responsivity at the UV region is linked to the energy gap of the NiO which is near to this wavelength, while the lower responsivity is related to the energy gap of the Si layer which is match to the NIR wavelength. When the energy of the incident photons was lower than the energy gap of NiO material, the photons will pass through the $\mathrm{NiO}$ and be absorbed by the silicon layer which will produce electron-hole pairs in the silicon layer. For this situation, the transport of electron-hole pairs will be affected by the built-in electric field which exists at the interface between $\mathrm{Ni}$ and $\mathrm{Si}$. Hence the obtained device can operate in two regions, but it is effective for UV region detectors. At the visible region, the responsivity is decreased, this is due to there is not sufficient energy to establish electron-hole pairs. The responsivity was calculated by the following equation [54]:

$$
\text { Responsivity }=\frac{I_{p h}-I_{d}}{P_{o p}}
$$

4

Where, $\mathrm{I}_{\mathrm{ph}}$ is the current under illumination, $\mathrm{I}_{\mathrm{d}}$ is the current under the dark case, and $\mathrm{P}_{\mathrm{op}}$ is the optical power of the photon source.

The most important factors for the photodetector device are repeatability and the speed of detection. Figure 8 demonstrates the photocurrent as a function of time at a reverse bias of $-1.5 \mathrm{~V}$ for the Ni sample that deposited on Si substrate at a distance of $25 \mathrm{~cm}$. The switching test was tested under a $365 \mathrm{~nm}$ wavelength LED source. The trend of this measurement shows good reproducibility and almost high stability for the UV detector device. It can be observed from the figure that, the five-cycle has rapid response time when the UV light is switched on and also rapid recovery time when the light is switched off.

The response and recovery times of the $\mathrm{Ni} / \mathrm{Si}$ device are based on the oxygen adsorption and desorption rates process. Before the illumination (dark case), the oxygen molecules were adsorbed on the surface of the sample [55]. These molecules will restrict free electrons, hence oxygen species will form $[56,57]$. According to this process, the conductivity decreased, and the depletion zone was established near the surface [58]. The lowest conductivity can result from the establishment of a high amount of species oxygen on the 
surface of the material which extends the band bending [59]. The second step is when the material is illuminated by UV light which has energy higher than the band gap of the material, this is leading to the generation of electron-hole pairs. Then, by combining the holes created by oxygen species on the surface, the oxygen species were released [60]. At the same moment, the free electrons will accumulate on the electrodes. Therefore, the life time of the created electrons will rise [61]. Because Ni material has hole carriers, the conductivity will rise [62]. The main reason behind the high photoresponse and quick response and recovery times is connected to the porous like structure which will make the detector device able to adsorb more oxygen molecules, (as shown from FESEM for the sample that deposited with a distance between the nozzle and the substrate is $25 \mathrm{~cm}$ ), and also the presence of nanoparticles beside these microstructures made the material possess high surface area. These two effective parameters act as an antireflective surface which guides the photons to generate a high amount of electron-hole pairs [63].

Figure 9 reveals an enlarged image of one cycle from Figure 8, that presents the response and the recovery times when the UV light switched ON and OFF, respectively. The response time ( $\tau_{\text {res }}$. ) is the duration that is required to increase the current from $10-90 \%$ before it reaches the saturation point. Whereas the recovery time $\left(\tau_{\text {rec }}\right.$. $)$ is the time that current is required to decrease from $90-10 \%$ before it reaches the reference point. As appeared from the figure, the response time is $0.375 \mathrm{~s}$ and the recovery time is $0.791 \mathrm{~s}$. In order to show the contrast between the current work and the other previous works, Table 2 lists the comparison of the performance of the photodetector based on $\mathrm{NiO}$ material.

Table 2

Comparison with prior photodetectors based on un-doped and doped NiO.

\begin{tabular}{|c|c|c|c|c|c|c|c|c|c|}
\hline Detector & $\begin{array}{l}\text { Method of } \\
\text { deposition }\end{array}$ & Morphology & $\lambda(\mathrm{nm})$ & $\begin{array}{l}\text { Power } \\
\text { density } \\
\left(\mathrm{mW} / \mathrm{cm}^{2}\right.\end{array}$ & $\begin{array}{l}\text { Bias } \\
\text { voltage } \\
\text { (V) }\end{array}$ & $\begin{array}{l}\text { Response } \\
\text { time (s) }\end{array}$ & $\begin{array}{l}\text { Recovery } \\
\text { time (s) }\end{array}$ & Responsivity & Ref. \\
\hline $\begin{array}{l}\mathrm{p}- \\
\mathrm{PNZO} / \mathrm{p}- \\
\mathrm{NiO} / \mathrm{n}^{-} \\
\mathrm{AZO}\end{array}$ & $\begin{array}{l}\text { Spray } \\
\text { Pyrolysis } \\
\text { method }\end{array}$ & - & 365 & 4 & 3 & 10.8 & 8.4 & $5.53(\mathrm{~A} / \mathrm{W})$ & [64] \\
\hline $\mathrm{TiO}_{2} / \mathrm{NiO}$ & $\begin{array}{l}\text { Hydrothermal } \\
\text { method }\end{array}$ & $\begin{array}{l}\mathrm{TiO}_{2} \\
\text { nanowell/NiO } \\
\text { mesoporous } \\
\text { nanosheet }\end{array}$ & 350 & 1.2 & 0 & 12 & 7.1 & $0.042(\mathrm{~A} / \mathrm{W})$ & [65] \\
\hline $\mathrm{NiO} / \mathrm{ZnO}$ & Electrospun & nanofiber & 350 & $75 \mathrm{~W}$ & 0 & 7.5 & 4.8 & $0.415 \mathrm{~mA} / \mathrm{W}$ & [66] \\
\hline $\begin{array}{l}\mathrm{p}-\mathrm{NiO} / \mathrm{n}- \\
\mathrm{Si}\end{array}$ & hydrothermal & nanoflakes & 450 & $2.5 \mathrm{~mW}$ & -3 & 6.18 & 1.83 & - & [25] \\
\hline $\mathrm{NiO} / \mathrm{Si}$ & Spin coating & nanosheets & 385 & 1.1 & -1 & 0.5 & 0.526 & $156.3 \mu \mathrm{A} / \mathrm{W}$ & [67] \\
\hline $\begin{array}{l}\text { n-Si } \\
(111) / p- \\
\mathrm{NiO}\end{array}$ & hydrothermal & nanosheets & $\begin{array}{l}350- \\
600 \mathrm{~nm}\end{array}$ & 0.5 & -2 & $\nabla 30 \mathrm{~ms}$ & $\nabla 30 \mathrm{~ms}$ & $0.43 \mathrm{~mA} / \mathrm{W}$ & [68] \\
\hline $\begin{array}{l}\mathrm{p}-\mathrm{NiO} / \mathrm{n}- \\
\mathrm{Si}\end{array}$ & sputtering & nanoparticles & 318 & - & -0.5 & - & - & $4.8 \mathrm{~A} / \mathrm{W}$ & [69] \\
\hline $\begin{array}{l}\text { p-NiO/n- } \\
\mathrm{Si}(100)\end{array}$ & $\begin{array}{l}\text { Spray } \\
\text { pyrolysis }\end{array}$ & $\begin{array}{l}\text { Mixed of } \\
\text { nanoparticles } \\
\text { and porous } \\
\text { microstructures }\end{array}$ & 365 & 2 & -1.5 & 0.375 & 0.791 & $6.5 \mathrm{~mA} / \mathrm{W}$ & $\begin{array}{l}\text { Current } \\
\text { work }\end{array}$ \\
\hline
\end{tabular}

\section{Conclusion}

Nickel Oxide material was sprayed on $\mathrm{n}$-(100) silicon substrate by spray pyrolysis method successfully. The effect of the distance between the nozzle and the substrate has been well studied. XRD and EDX analyses confirmed the presence of NiO and Si without any other unwanted materials. A high degree of crystallinity and stoichiometric has been obtained for the sample with $25 \mathrm{~cm}$ distance, as proved by the reduction of oxygen element from EDX analysis. Also, this sample showed a surface with a mixture of micro and nano particles, and the microparticles have a porous like structure. The photoluminescence revealed spectra that have three main peaks lie in UV and visible regions of the electromagnetic spectrum which confirmed the near band edge emission and the existence of the defects, respectively. The Current-Voltage characteristics depicted well rectifying properties with a higher photoresponse under $365 \mathrm{~nm}$ compared with the dark current. The photoresponse of this device is connected to the movement of electrons to the n-Silicon and holes to p-Nickel 
Oxide by a high built-in electric field. Furthermore, the current-time characteristics showed swift response and recovery times toward UV light with constant reproducibility and high stability. The tested detector has been done under a low bias voltage of 1.5 Volt. Subsequently, the obtained device can be used in optoelectronic detectors.

\section{Declarations}

\section{Acknowledgment}

The authors would like to thank the department of physics, University of Tehran, Tehran, Iran, and thin films lab., department of physics, University of Mustansiriyah, Baghdad, Iraq.

\section{References}

[1] H.Y. Chen, K.W. Liu, X. Chen, Z.Z. Zhang, M.M. Fan, M.M. Jiang, X.H. Xie, H.F. Zhao, D.Z. Shen, Realization of self-powered ZnO MSM UV photodetector with high responsivity using asymmetric pair of Au electrodes. J. Mater. Chem. C 2, 9689 (2014)

[2] G. Rajan, A. Kaur, H.K. Paliwal, V. Yadav, Gupta, M. Tomar, Plasmonic assisted enhanced photoresponse of metal nanoparticle loaded ZnO thin film ultraviolet photodetectors. J. Phys. D 47, 425102 (2014)

[3] Abed, Husam R., et al. "Efficient $\mathrm{SnO} 2 / \mathrm{CuO}$ /porous silicon nanocomposites structure for $\mathrm{NH} 3$ gas sensing by incorporating CuO nanoparticles." Optical and Quantum Electronics 51.10 (2019): 1-13.

[4] Yousif, Ali A., Alwan M. Alwan, and Husam R. Abed. "Optimizing of macro porous silicon morphology for creation of $\mathrm{SnO} / \mathrm{CuO}$ nanoparticles." AIP Conference Proceedings. Vol. 2213. No. 1. AIP Publishing LLC, 2020.

[5] Abed, Husam R., et al. "Efficient, fast response, and low cost sensor for $\mathrm{NH} 3$ gas molecules based on SnO 2: CuO/macroPSi nanocomposites." Applied Physics A 126.11 (2020): 1-15.

[6] Alwan, Alwan M., Husam R. Abed, and Rasha Bashar Rashid. "Enhancing the temporal response of modified porous silicon-based CO gas sensor." Solid-State Electronics 181 (2021): 108019.

[7] Khudadad, Ameer I., Ali A. Yousif, and Husam R. Abed. "Effect of heat treatment on WO3 nanostructures based NO2 gas sensor lowcost device." Materials Chemistry and Physics (2021): 124731.

[8] Chala, Slimane, et al. "Extraction of ZnO thin film parameters for modeling a ZnO/Si solar cell." Energy 164 (2018): 871-880.

[9] Ozel, Kenan, et al. "A route towards enhanced UV photo-response characteristics of SnO2/p-Si based heterostructures by hydrothermally grown nanorods." Journal of Alloys and Compounds 849 (2020): 156628.

[10] Alsultany, Forat H., Z. Hassan, and Naser M. Ahmed. "A high-sensitivity, fast-response, rapid-recovery UV photodetector fabricated based on catalyst-free growth of ZnO nanowire networks on glass substrate." Optical Materials 60 (2016): 30-37.,

[11] Liu, Sisi, et al. "Self-assembled Al nanostructure/ZnO quantum dot heterostructures for high responsivity and fast UV photodetector." Nano-micro letters 12 (2020): 1-13.,

[12] Alam, Md Jawaid, et al. "Enhancing responsivity and detectivity in broadband UV-VIS photodetector by ex-situ UV-ozone annealing technique." Superlattices and Microstructures 137 (2020): 106333.

[13] Ning, Yi, et al. "Novel transparent and self-powered UV photodetector based on crossed ZnO nanofiber array homojunction." Small 14.13 (2018): 1703754.

[14] Chahrour, Khaled M., F. K. Yam, and Raed Abdalrheem. "High-performance UV photodetector of anodic rutile TiO2 nanotube arrays." Materials Letters 248 (2019): 161-164.,

[15] Parida, Bhaskar, et al. "Nanostructured-NiO/Si heterojunction photodetector." Materials science in Semiconductor Processing 71 (2017): 29-34. 
16]] Marimuthu, G., et al. "Influence of twin boundaries on the photocurrent decay of nanobranch and dense-forest structured SnO2 UV photodetectors." Superlattices and Microstructures 128 (2019): 181-198.,

[17] Yadav, PV Karthik, et al. "Oxygen partial pressure dependent UV photodetector performance of WO3 sputtered thin films." Journal of Alloys and Compounds 816 (2020): 152565.

[18] Gomaa, M. M., et al. "Effect of precursor solutions on the structural and optical properties of sprayed NiO thin films." Materials Science in Semiconductor Processing 64 (2017): 32-38.,

[19] Singh, S. D., et al. "Studies on structural and optical properties of pulsed laser deposited NiO thin films under varying deposition parameters." Materials Science in Semiconductor Processing 66 (2017): 186-190.,

[20] Gomaa, M. M., et al. "Structural and optical properties of nickel oxide thin films prepared by chemical bath deposition and by spray pyrolysis techniques." Journal of Materials Science: Materials in Electronics 27.1 (2016): 711-717.

[21] Ji, Yuexing, et al. "Ultraviolet photodetectors using hollow p-CuO nanospheres/n-ZnO nanorods with a pn junction structure." Sensors and Actuators A: Physical 304 (2020): 111876.,

[22] Gunasekaran, S., et al. "Fabrication of high-performance SiO2@ p-CuO/n-Si core-shell structure based photosensitive diode for photodetection application." Surfaces and Interfaces 20 (2020): 100622.,

[23] Reddy, K. Chandra Sekhar, et al. "One-step fabrication of 1D p-NiO nanowire/n-Si heterojunction: Development of self-powered ultraviolet photodetector." Applied Surface Science 513 (2020): 145804.,

[24] Thongma, Sattra, et al. "UV enhanced white-light response based on p-Si/n-ZnO nanorod heterojunction photosensor." Sensors and Actuators A: Physical 296 (2019): 324-330.

[25] Jayalakshmi, G., et al. "Fabrication of p-NiO nanoflakes/n-Si (100) heterojunction architecture for high sensitive photodetectors." Journal of Materials Science: Materials in Electronics 30.7 (2019): 6811-6819.

[26] Choi, Jeong-M., and Seongil Im. "Ultraviolet enhanced Si-photodetector using p-NiO films." Applied Surface Science 244.1-4 (2005): 435-438.

[27] Zhang, Dongyuan, Shinji Nozaki, and Kazuo Uchida. "NiO/Si heterostructures formed by UV oxidation of nickel deposited on Si substrates." Journal of Vacuum Science \& Technology B, Nanotechnology and Microelectronics: Materials, Processing, Measurement, and Phenomena 32.3 (2014): 031202.

[28] Wang, Yali, et al. "Facet-dependent photocatalytic performance of NiO oriented thin films prepared by pulsed laser deposition." Physica B: Condensed Matter 457 (2015): 194-197

[29] Krunks, M., et al. "Deposition of p-type NiO films by chemical spray pyrolysis." Vacuum 107 (2014): 242-246

[30] Sun, Jingsong, et al. "Inverted perovskite solar cells with high fill-factors featuring chemical bath deposited mesoporous NiO hole transporting layers." Nano Energy 49 (2018): 163-171

[31] Chen, S. C., et al. "Characterization and properties of NiO films produced by rf magnetron sputtering with oxygen ion source assistance." Thin Solid Films 572 (2014): 51-55

[32] Goumri-Said, Souraya, et al. "Europium incorporation dynamics within NiO films deposited by sol-gel spin coating: experimental and theoretical studies." Materials Research Bulletin 118 (2019): 110525

[33] Habubi, Nadir F., et al. "Annealing time effect on nanostructured n-ZnO/p-Si heterojunction photodetector performance." Surface Review and Letters 22.02 (2015): 1550027

[34] Fasaki, I., et al. "Structural, electrical and mechanical properties of NiO thin films grown by pulsed laser deposition." Applied Surface Science 257.2 (2010): 429-433.

[35] Gokul, B., et al. "Structural and dielectric properties of NiO nanoparticles." Journal of non-crystalline solids 363 (2013): 161-166. 
[36] Arif, Mohd, et al. "Influence of interparticle interaction on the structural, optical and magnetic properties of $\mathrm{NiO}$ nanoparticles." Physica B: Condensed Matter 552 (2019): 88-95.

[37] Yousaf, Sheraz, et al. "Tuning the structural, optical and electrical properties of NiO nanoparticles prepared by wet chemical route." Ceramics International 46.3 (2020): 3750-3758.

[38] Chtouki, T., et al. "Comparison of structural, morphological, linear and nonlinear optical properties of NiO thin films elaborated by spin-coating and spray pyrolysis." Optik 128 (2017): 8-13.

[39] Alwan, Alwan M., Ali A. Yousif, and Husam R. Abed. "High sensitivity and fast response at the room temperature of SnO2: CuO/PSi nanostructures sandwich configuration NH3 gas sensor." AIP Conference Proceedings. Vol. 2190. No. 1. AIP Publishing LLC, 2019.

[40] Othman, A. A., et al. "Sonochemically synthesized ZnO nanosheets and nanorods: Annealing temperature effects on the structure, and optical properties." Ceramics International 43.1 (2017): 527-533

[41] Yousif, Ali A., and Ameer I. Khudadad. "Effects of Annealing Process on the W03 Thin Films Prepared by Pulsed Laser Deposition." IOP Conference Series: Materials Science and Engineering. Vol. 745. No. 1. IOP Publishing, 2020.

[42] Alwan, Alwan M., Husam R. Abed, and Ali A. Yousif. "Effect of the deposition temperature on ammonia gas sensing based on SnO 2/porous silicon." Plasmonics 16.2 (2021): 501-509.

[43] Yousif, Ali A., Reeman M. Hathal, and Husam R. Abed. "The Effectiveness of Decorating Antimony on the Structural, Optical, and Electrical Characteristics of SnO2 Nanowires." Journal of Electronic Materials (2021): 1-11

[44] Manikandan, A., J. Judith Vijaya, and L. John Kennedy. "Comparative investigation of NiO nano-and microstructures for structural, optical and magnetic properties." Physica E: Low-Dimensional Systems and Nanostructures 49 (2013): 117-123

[45] Zhao, Jinbo, Lili Wu, and Ke Zou. "Fabrication of hollow mesoporous NiO hexagonal microspheres via hydrothermal process in ionic liquid." Materials Research Bulletin 46.12 (2011): 2427-2432

[46] Xu, Chunju, et al. "Hydrothermal synthesis of $\beta-\mathrm{Ni}(\mathrm{OH}) 2$ platelets and their thermal conversion to NiO." Journal of Materials Science: Materials in Electronics 25.9 (2014): 3716-3720

[47] Nabi, Ghulam, et al. "Structural, Optical, and Magnetic Properties of Pure and Vanadium-Doped NiO Microstructures for Spintronics Applications." Journal of Superconductivity and Novel Magnetism (2020): 1-6.

[48] Tamura, Kenki, et al. "Ni-based composite microstructures fabricated by femtosecond laser reductive sintering of $\mathrm{NiO} / \mathrm{Cr}$ mixed nanoparticles." Japanese Journal of Applied Physics 56.6S1 (2017): 06GN08.

[49] S. Majumder, S. Bhattacharjee, C.K. Ghosh, NiO/Ag heterostructure: enhanced UV emission intensity, exchange interaction and photocatalytic activity. RSC Adv. 6, 56503-56510 (2016)

[50] Jayalakshmi, K. Saravanan, J. Pradhan, P. Magudapathy, B.K. Panigrahi, Facile synthesis and enhanced luminescence behavior of ZnO: reduced graphene oxide(Rgo) hybrid nanostructure. J. Lumin. 203, 1-6 (2018)

[51] Mousa, Ali M., Raid A. Ismail, and Mustafa H. Amin. "Hybrid p-Au@ PbI2/n-Si heterojunction photodetector prepared by pulsed laser ablation in liquid." Optik 183 (2019): 933-941.

[52] Khayatian, A., et al. "Effect of annealing process in tuning of defects in ZnO nanorods and their application in UV photodetectors." Optik 127.11 (2016): 4675-4681

[53] C. Soci, A. Zhang, B. Xiang, S.A. Dayeh, D.P.R. Aplin, J. Park, X.Y. Bao, Y.H. Lo, D. Wang, ZnO Nanowire UV Photodetectors with high internal gain, Nano Lett. 7 (2007) 1003-1009.

[54] Jwied, Duha H., Uday M. Nayef, and Falah AH Mutlak. "Synthesis of C: Se (core: shell) nanoparticles via laser ablation on porous silicon for photodetector application." Optik 231 (2021): 166493 
[55] J. Cheng, Y. Zhang, R. Guo, ZnO microtube ultraviolet detectors, Journal of Crystal Growth, 310 (2008) 57-61.

https://doi.org/10.1016/j.jcrysgro.2007.08.034

[56] R. Azimirad, A. Khayatian, M.A. Kashi, S. Safa, Electrical investigation and ultraviolet detection of ZnO nanorods encapsulated with ZnO and Fe-doped ZnO layer, Journal of sol-gel science and technology, 71 (2014) 540-548. https://doi.org/10.1007/s10971-014-3406-5

[57] Z. Lim, Z. Chia, M. Kevin, A. Wong, G. Ho, A facile approach towards ZnO nanorods conductive textile for room temperature multifunctional sensors, Sensors and Actuators B: Chemical, 151 (2010) 121- 126. https://doi.org/10.1016/j.snb.2010.09.037.

[58] J. Kar, S. Das, J. Choi, Y. Lee, T. Lee, J. Myoung, Fabrication of UV detectors based on ZnO nanowires using silicon microchannel, Journal of Crystal Growth, 311 (2009) 3305-3309. https://doi.org/10.1016/j.jcrysgro.2009.04.005

[59] Dhara, Soumen, and P. K. Giri, Enhanced UV photosensitivity from rapid thermal annealed vertically aligned ZnO nanowires, Nanoscale research letters, 6 (2011) 504. https://doi.org/10.1186/1556-276X-6- 504

[60] M.-W. Chen, C.-Y. Chen, D.-H. Lien, Y. Ding, J.-H. He, Photoconductive enhancement of single ZnO nanowire through localized Schottky effects, Optics Express 18 (2010) 14836-14841. https://doi.org/10.1364/OE.18.014836

[61] S. Safa, S. Mokhtari, A. Khayatian, R. Azimirad, Improving ultraviolet photodetection of ZnO nanorods by Cr doped ZnO encapsulation process, Optics Communications, 413 (2018) 131-135. https://doi.org/10.1016/j.optcom.2017.12.038

[62] Y. - L. Chu, L. -W. Ji, Y. - J. Hsiao, H. -Y. Lu, S. - J. Young, I. -T. Tang, T. - T. Chu, X. - J. Chen, Fabrication and Characterization of NiDoped ZnO Nanorod Arrays for UV Photodetector Application, Journal of The Electrochemical Society, 167 (2020) 067506. https://doi.org/10.1149/1945-7111/ab7d43

[63] D.S. Tsai, C.A. Lin, W.C. Lien, H.C. Chang, Y.L. Wang, J.H. He, Ultra-highresponsivity broadband detection of Si metalesemiconductoremetal Schottky

photodetectors improved by ZnO nanorod arrays, ACS Nano 5 (2011) 7748e7753.

[64] R. Amiruddin, M.S. Kumar, Role of p-NiO electron blocking layers infabrication of (PN): ZnO/Al: znO UV photodiodes, Curr. Appl. Phy. 16 (9)(2016) 1052-1061.

[65] L. Zheng, et al., Large scale: highly efficient and self-powered UVphotodetectors enabled by all-solid-state n-TiO 2 nanowell/pNiOmesoporous nanosheet heterojunctions, J. Mater. Chem. C 4 (42) (2016)10032-10039.

[66] Z. Zhang, Y. Ning, X. Fang, From nanofibers to ordered ZnO/NiO heterojunction arrays for self-powered and transparent UV photodetectors, J. Mater. Chem. C. 7 (2019) 223-229,

[67] S. Parida, M. Kim, S. Oh, M. Jung, J.H. Baek, H. Ryou, Kim, Nanostructured-NiO/Si heterojunction photodetector. Mater. Sci. Semicond. Process. 71, 29-34 (2017)

[68] Y. Zhang, T. Ji, W. Zhang, G. Guan, Q. Ren, K. Xu, X. Huang, R. Zou, J. Hu, A self-powered broadband photodetector based on an n$\mathrm{Si}(111) / \mathrm{p}-\mathrm{NiO}$ heterojunction with high photosensitivity and enhanced external quantum efficiency. J. Mater. Chem. C 5, 12520 (2017)

[69] Hammadi, Oday A., Mohammed K. Khalaf, and Firas J. Kadhim. "Farbication of UV photodetector from nickel oxide nanoparticles deposited on silicon substrate by closed-field unbalanced dual magnetron sputtering techniques." Optical and Quantum Electronics 47.12 (2015): 3805-3813.

\section{Figures}




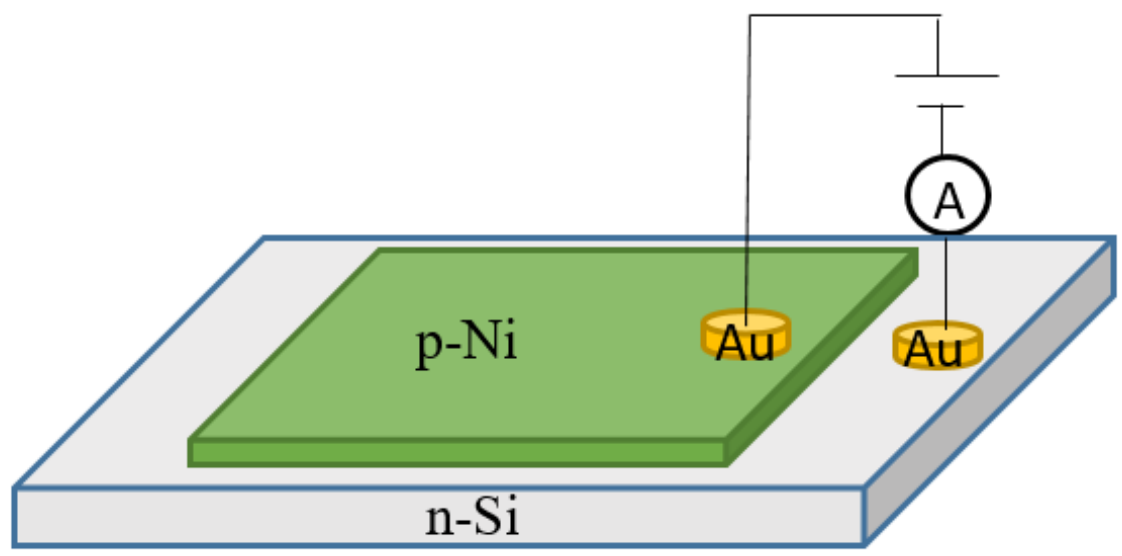

Figure 1

Photodetector device configuration.

Figure 2

XRD patterns of NiO deposited at different distances between the nozzle and the Si substrate. 


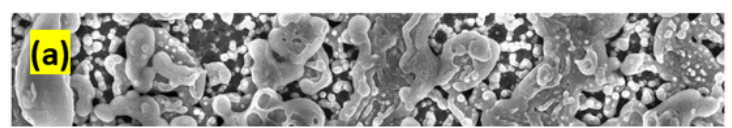

\section{Figure 3}

FESEM images of NiO deposited on Si at different distances between the nozzle and the substrate. 

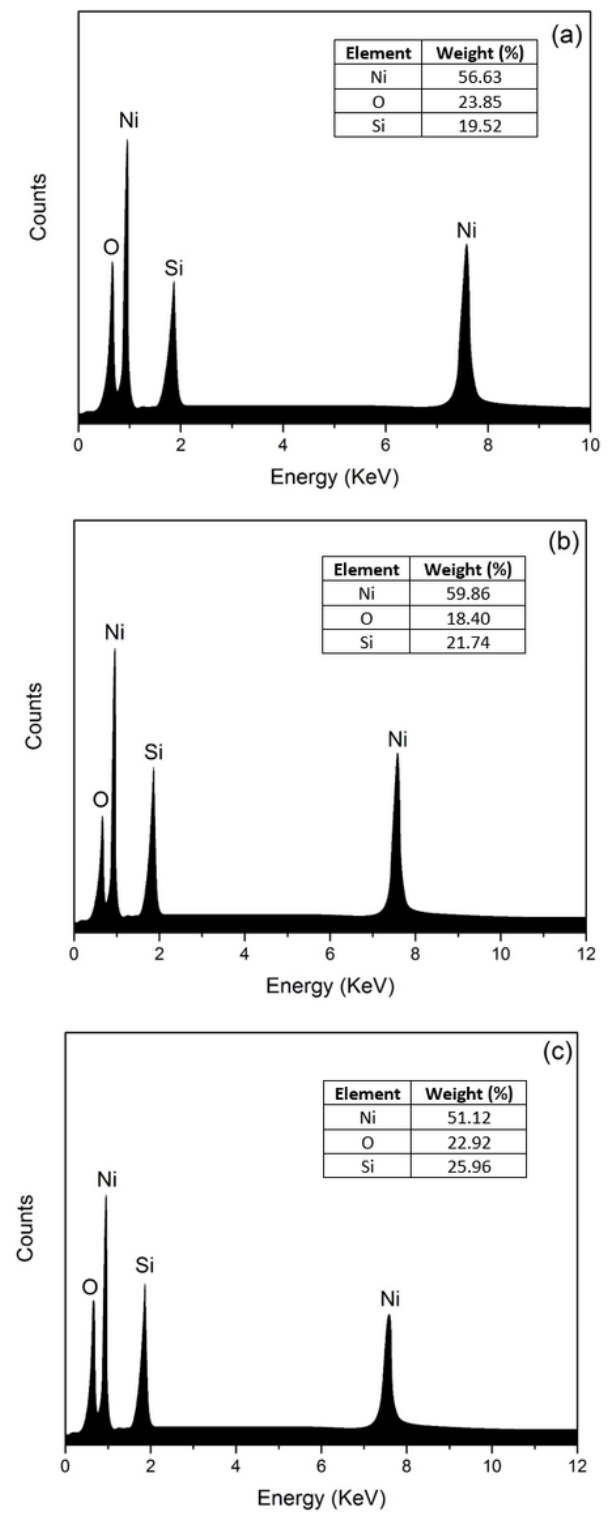

Figure 4

EDX for chemical elemental analysis for NiO deposited at different distances between the nozzle and the Si substrate (a) $20 \mathrm{~cm}$, (b) 25 $\mathrm{cm}$, and (c) $30 \mathrm{~cm}$. 


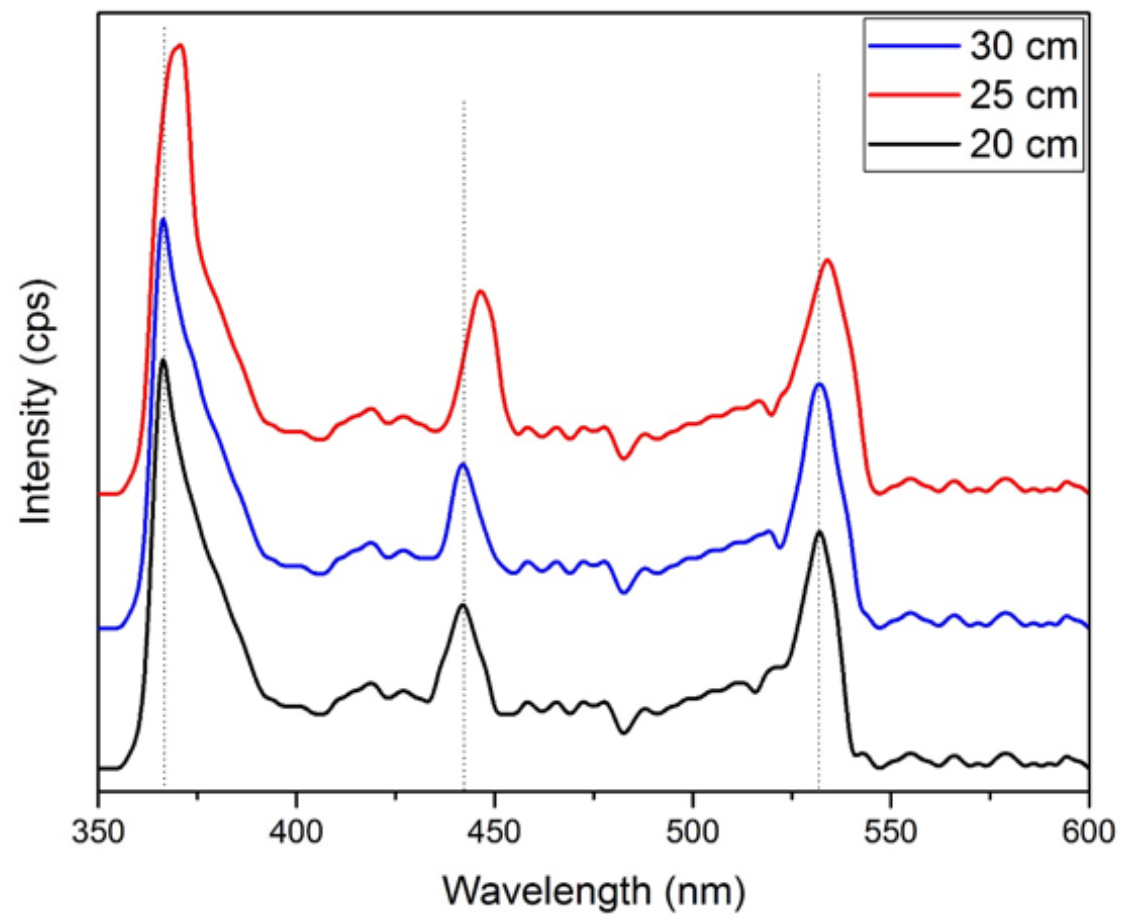

Figure 5

PL spectra of NiO deposited on Si substrate at different distances.

Figure 6

Current-Voltage characteristics of $\mathrm{NiO} / \mathrm{Si}$ at different distances under dark and illumination cases.

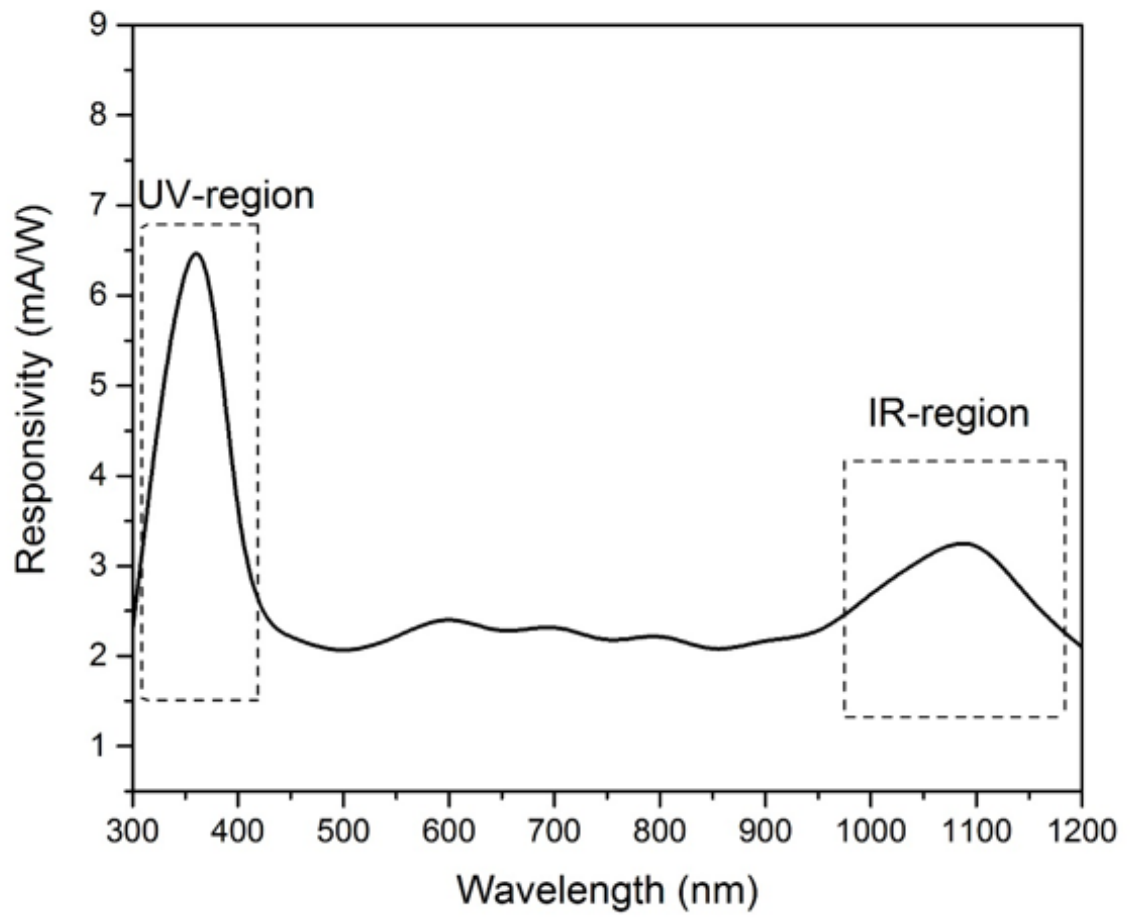

Figure 7

Page 14/16 
Responsivity as a function of wavelength of $\mathrm{NiO} / \mathrm{Si}$ at different distances.

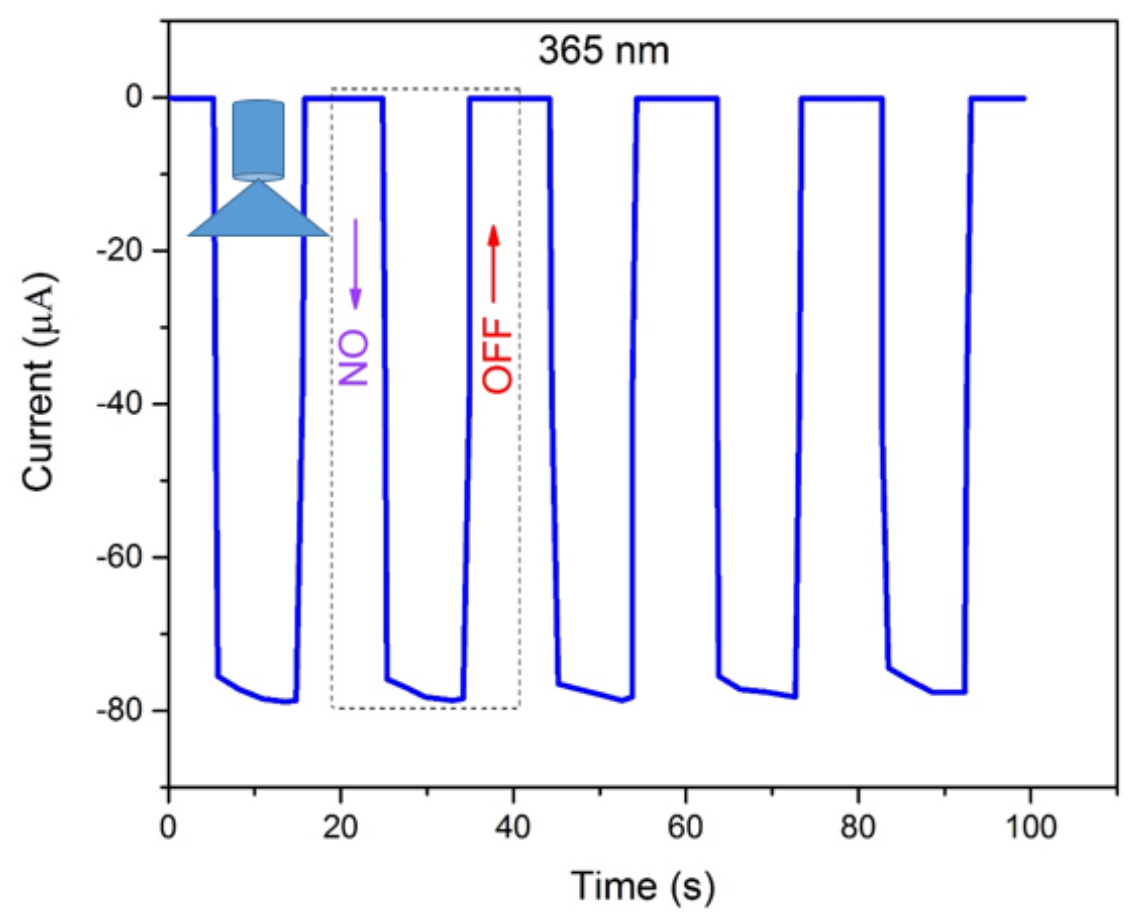

Figure 8

Current-Time characteristics of $\mathrm{NiO} / \mathrm{Si}$ at different distances under UV illumination at a constant voltage of 1.5 Volt. 

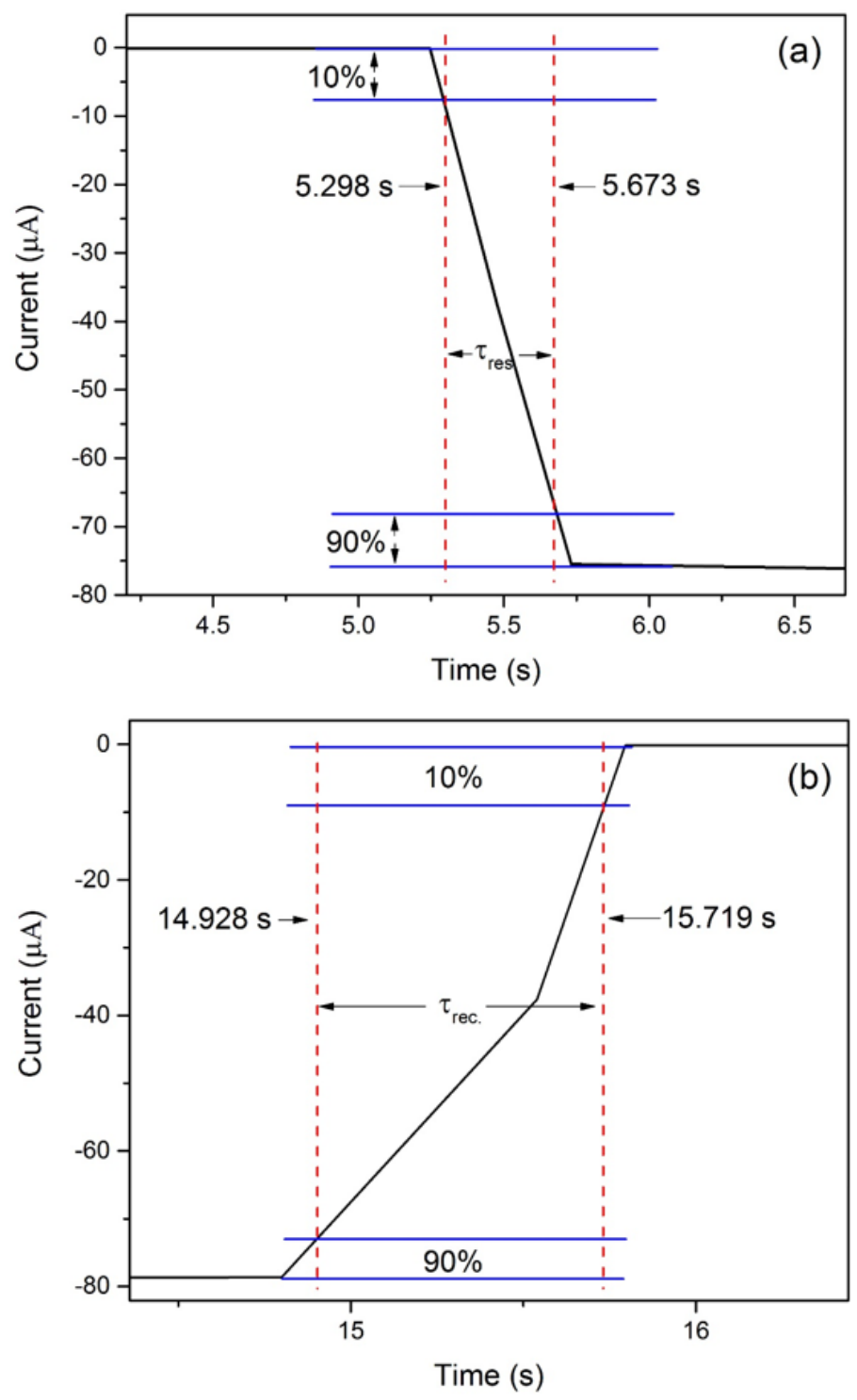

Figure 9

Enlarged view of response and recovery times for one cycle. 